\title{
The velocity of meteoroids: a historical review
}

\author{
I. P. Williams \\ Astronomy Unit, Queen Mary, University of London, Mile End Road, London E1 4NS, UK \\ Received: 16 September 2003 - Published in Atmos. Chem. Phys. Discuss.: 7 January 2004 \\ Revised: 16 March 2004 - Accepted: 17 March 2004 - Published: 23 March 2004
}

\begin{abstract}
Determining the velocity of meteoroids as they enter the Earth's atmosphere is very important since the value is fundamental in calculating the orbit of the meteoroid and hence eventually its origin. We describe early attempts at this determination and highlight problems that exist today.
\end{abstract}

\section{Introduction}

Unless we live in a very special and unique time, meteors must have been witnessed by the human race since antiquity. Records of their appearances, described using terms like many falling stars date back for at least two millennia (see Hasegawa, 1993). However, serious scientific analysis is much more recent and only dates back for a tenth of this time-span, or about two hundred years. One of the earliest measurement of any meteor property was by Benzenberg and Brandeis (1800), who were at the time students at Göttingen University. They observed the same set of meteors from two different locations and through parallax found the height to be about $90 \mathrm{~km}$ (This, as it turned out, was a remarkably accurate determination of the typical height of meteors). The angular velocity of a meteoroid can be determined from the time taken to cover the visible trail, so that with the height determined, both velocity and position became known, enough information to calculate the Keplerian orbit. Obtaining the orbit of a meteoroid is in fact very import, for knowledge of it makes it possible for us to identify the probable parent body. The Leonid storms of 1799, 1833 and 1866 helped Adams (1867), LeVerrier (1867) and Schiaparelli (1867) to conclude that the orbit of the Leonid meteoroid stream was remarkably similar to that of a comet, newly discovered in 1861 , that we now know as 55P/Tempel-Tuttle. This discovery naturally led to the proposition that comets and meteor showers

Correspondence to: I. P. Williams

(i.p.williams@qmul.ac.uk) were very closely related. In fact, without some record of the event, determining the time taken for the meteor to cover a given distance was more a matter of guessing than real measuring. In time, photography became possible, so that the trail can now be easily measured. However, the time taken is still undetermined, it could be any quantity smaller than the exposure time. To overcome this problem, two new devices were produced, the rotating shutter and the rocking mirror. In the first, an obstruction rotates at a known rate in front of the camera lens, in effect blocking off the light from the photographic plate at given time intervals. This leads to the trail on the photograph having a series of breaks in it, with the time interval between breaks known. In the second, the mirror which reflects light into the camera oscillates, or rocks, at a known rate, causing the light to fall on slightly different parts of the photographic plate and leading to a wave-like trail rather than a straight one, with the time interval between given points on the wave being known. The first method is still in use today, though with video equipment and charge coupled devices (CCD's) replacing the photographic plate. The principle however remains the same, the time taken to cover a specific distance is obtained. In 1895 Weinek (1886) obtained the first ever photograph of a meteor, though unfortunately his attempt to obtain multi-station photography failed. Photographic work on meteors was also carried out by Elkin (1899) and he obtained the first accurate determination of meteor velocities (Elkin, 1900).

As soon as semi-reliable methods for velocity determination became available, a controversy emerged, namely whether or not hyperbolic meteoroids existed. In practice, this is taken to mean meteors with a heliocentric velocity in excess of the escape velocity $\left(42.13 \mathrm{~km} \mathrm{~s}^{-1}\right)$. Any meteor with a velocity upon entry into the atmosphere in excess of $72.75 \mathrm{~km} \mathrm{~s}^{-1}$ must satisfy the above condition. This controversy is still with us, but first emerged with the publication of a catalogue by von Niessel and Hoffmeister (1925) in which $79 \%$ of meteoroids were hyperbolic. Öpik (1932) claimed 
that clouds of comets existed at large distances around the sun (pre-dating Öort by nearly 20 years) and other stars and that stellar perturbations could cause these to escape. Öpik became a firm defender of the hyperbolic meteoroid theory, and took part in an expedition to Arizona to measure meteor velocities (Shapely et al., 1932). The results were initially published in 1934 (Öpik, 1934) while Öpik (1940) again gave an analysis of 1436 meteor velocities, claiming that $60 \%$ were hyperbolic. The opposite view was taken by Whipple (1938, 1940) and Porter (1942), who claimed that most hyperbolic velocities were measurement errors. As time passed, more surveys produced additional results, using more meteoroids, for example McCrosky and Possen (1961) measured 2529 meteors and found only $10.9 \%$ hyperbolic velocities, a number that could be accommodated within the error bars. Eventually, Öpik (1969) conceded that his early measurements had been in error.

All of the above results were obtain through photographing fairly bright, that is, large meteors. There are many more small meteoroids and, statistically, the reliability of the results would improve with additional data. To extend our knowledge to the small particle end of the interplanetary dust complex, science had to wait until the birth of radio astronomy.

\section{The early days of radio astronomy}

Early radio transmissions made use of the fact the electromagnetic waves could be reflected from a conducting surface, and early work by Appleton and Barnett (1925), Breit and Tuve $(1925,1926)$ established the existence of a conducting ionized layer in the upper atmosphere. Appleton (1930) discover that there was a sudden increase in the ionization during the night, concluding that "there was some agent present which can influence the dark side of the Earth". Nagaoka (1929) had suggested that meteors could affect the propagation of radio waves, but this idea seems not to have received much attention at the time. These changes in the ionization level of the ionosphere were causing serious problems for radio communications and Skellett was employed by Bell labs, his main duties being to find ways of improving transmission quality. One school of thought suggested that cosmic rays were affecting the ionosphere, hence causing the problem. Skellett $(1931,1932,1935)$ (apparently ignorant of Nagaoka's work) independently suggested instead that meteors might be the culprits. In a paper immediately following Skellet's second paper referred to above, Schafer and Goodall (1932) had found very disturbed conditions in the ionosphere during the Leonid shower of 1931. At about the same time Jansky had developed a rotating aerial in order to try to locate the source of static noise in transmissions, which was eventually shown to be from a source near the center of the Milky Way. Perhaps because of this, little seems to have been done for a number of years to either verify or disprove Skellett's suggestion that meteors were the culprits.

On 12 February 1942, an incident took place which had no direct bearing whatsoever on our meteor story, namely the sailing of the two warships, Schanhorst and Gneisnau from Brest to Germany undetected by the British Navy. Books have been written about the incident and how it was achieved, but this communication is not the place to investigate this interesting story. The British Costal radar system had however been jammed throughout the incident and, in consequence, The Army Operational Research Group was order to give top priority to solving this problem of radar jamming. The director of the group (Schonland) turned to Hey to carry this out. Two weeks later, further jamming was reported, this time to the anti-aircraft gun-laying radars, but no enemy bombing attacks materialized. Hey (1942) (in a report that was to remain secret for many years) discovered that the jamming was in fact caused by solar outbursts rather than through any action on the part of the German military. It is an other case where radars were apparently being jammed that is relevant to the development of the meteor story. In 1944, Hey was involved in modifying the anti-aircraft radar system so that they could detect V2 rockets soon after launch in the hope that a few minutes warning could be given to the civilian population. A major problem encountered was the existence of a large number of transient radar echoes, resulting in many false alarms. Hey and Stewart (1946) proved that these transient echoes were from meteor trails because of the increase in echoes during the Quadrantid shower in January and the Lyrid shower in April. Final proof of the correctness of this meteor hypothesis came with the Giacobinid meteor storm of 1946, when 10000 echoes per hour were recorded instead of the usual 2 or so (Hey et al., 1947; Lovell et al., 1947). Hey and Stewart (1947) also identified the echo as being the "head echo', that is an echo caused through the specular reflection of the incident radio wave off the head of the meteor. McKinley and Millman (1949) showed that by the application of simple geometric principles, individual meteor orbits as well as meteor velocities could be determined using head-echoes provided three stations were used. At about the same time, Appleton and Naismith (1947) found that the wavelength of the returning echo was changed, and concluded that this was due to the Doppler Effect which provided a further method to determine meteor velocities.

Herlofson (1946) had suggested that the ionized trail behind a meteor should, in theory, produce the well known Fresnel diffraction pattern. This pattern was successfully observed by Ellyet and Davies (1948) who proceeded to measure velocities of daytime meteor showers (Ellyett, 1949), their existence having been demonstrated by Clegg et al. (1947). Davies became interested in the problem of the existence or otherwise of hyperbolic meteors mentioned earlier and made extensive use of the Jodrell Bank telescopes in an attempt to solve this problem. Unfortunately, Davies was not one of the World's prolific publishers and, though having 
completed observations, eventually of 13000 sporadic meteors by 1955, these were not published until 1960 (Davies and Gill, 1960). The vast majority were found to be on elliptical orbits and the number of hyperbolic meteors were small enough to be attributable to observing errors. As time progressed, more and more data became available and, by 1970, the radio data were producing a consistent picture where the hyperbolic fraction of sporadic meters were between 2 and $3 \%$. The data from visual observations of meteors still produced a much higher fraction, $10-25 \%$, though the total number of meteors observed was much smaller. Most astronomers considered the determined high velocities to be caused by errors, mainly as a result of the difficulty of measuring accurately the positions of faint meteors.

Thus, by this time the three standard methods of measuring meteor velocities by radio, namely geometric, Doppler shift and Fresnel diffraction, had been established and the general consensus was that hyperbolic meteors did not exist.

\section{The position now}

The basic techniques for measuring the velocities of visible meteors has remained the same since the 1950's, though methods other than photographic are now used for recording events. Unfortunately, the number of systematic surveys of meteors that are being carried out now is small and most of the recent observations concentrate on well-known streams, for example work by the Dutch Meteor Society (Betlem et al., 1997). The AKM system (Molau, 2001) does produce semi-automatic all year coverage, observing some 20000 meteors, but can not produce velocities as the same meteors are not observed from two locations. The European Fireball Network (Spurneý, 1997) does produce double station observations, but only of the brighter fireballs. Using TV double station observations, Ueda et al. (2001) found significant numbers of hyperbolic meteors. In the early part of the nineties, the dedicated meteor radar system AMOR (Baggaley et al., 1994) became operational and this is capable of measuring meteor speed in very large numbers (over 350000 to date). Results indicate that $10 \%$ of all ecliptic meteors are hyperbolic, with as much as $40 \%$ of meteors coming from an area within $15^{\circ}$ of a source with coordinates $(\lambda, \beta)$ of $260^{\circ}$, $-57^{\circ}$ being hyperbolic (Baggaley and Gallighan, 2001). At about the same time, the the large aperture radars at EISCAT (Pellinen-Wannberg and Wannberg, 1994) and at Arecebo (Zhou et al., 1995) began to regularly observe meteors. This significantly increased the numbers of measured meteors but also extend the range in size down to much smaller (i.e. $100 \mu \mathrm{m}$ radius) meteors than had hitherto been regularly observed. All found velocities that have a mean value higher than previously obtained and found significant numbers of hyperbolic meteors (Taylor et al., 1996; Janches et al., 2001).

\section{Summary and conclusions}

Thus we are back to the to where we were in the the 1930's, do hyperbolic meteors exist or not? Resolving this meteor velocity argument was one of the main motivations for holding this workshop and so it would not be correct for further discussion to take place here regarding current measurements. Other papers will throw light on this. I will simply restate the position, namely that two major problems exist:

1. The large aperture radars appear to measure higher mean velocities than conventional radars. At first sight, this could easily be resolved through observing the same meteor using two different techniques. Since meteors ablate at about $100 \mathrm{~km}$ above the ground, a difficulty in doing this is that both observing facilities have to be within about $100 \mathrm{~km}$ or so of each other in order that they can see the same meteor. Since the large aperture radars are large permanent fixtures, this means placing an optical facility close to the radar one. This has been tried by Pecan et al. (2001), but the problem is that radar detects far fainter (and hence more) meteors that the optical facility and it becomes very difficult to identify which of the radio meteors corresponds to an observed visible one. Indeed, in the above case, the similarity of the velocity measurement was used to help identification. A further problem is the very discrepant field of view, being less than $1^{\circ}$ for the large aperture radars, about $3^{\circ}$ for the classical meteor radars, something like $20^{\circ}$ for TV observations and something close to $90^{\circ}$ for visible observations.

The large aperture telescopes are only able to observe significant numbers of meteors because, on average, they detect much smaller sized meteors. To illustrate the problem, Pellinen-Wannberg (2001), observing with the large aperture EISCAT telescope at the height of the 1998 and 1999 Leonid storms, detected 10 meteors but none were Leonids.

We need to understand whether the discrepancy is because of differences in the reduction techniques and underlying assumptions, or do the smaller meteors genuinely have larger impact velocities with the Earth. If the second alternative is shown to be correct, we would also like to understand how this could be so.

2. A large number of meteors, using these new radars, are recorded as having velocities well in excess of the hyperbolic limit. Are these real, and if so, what is the source of these interstellar meteors. It is also worth pointing out that these hyperbolic meteors are (almost by definition since stream meteors are on bound orbits) part of the sporadic background. On the other had the vast majority of visible observations are carried out around predicted shower maximum times and so, almost all observed meteors are stream meteors. 
Acknowledgement. The author would like to thank EOARD for financial support through their Window On Science scheme which made attendance at the workshop and hence this publication possible.

Edited by: D. Janches

\section{References}

Adams, J. C.: On the orbit of the November Meteors, Mon. Not. R. astr. Soc., 27, 247-252, 1867.

Appleton, E. V.: On some measurements of the Equivalent height of the Atmospheric Ionized Layer, Proc. Roy. Soc., A126, 542-569, 1930.

Appleton E. V. and Barnett, M. A. F.: Local Reflection of Wireless Waves from the upper Atmosphere, Nature, 115, 333-334, 1925.

Appleton, E. V. and Naismith, R.: Radio detection of Meteor trails and allied phenomena, Proc. Roy. Soc., A59, 461-472, 1947.

Baggaley, W. J., Bennett, R. G. T., Steel, D. I., and Taylor, A. D.: Advanced Meteor Orbit Radar: AMOR, Qt. Jl. R. Astr. Soc., 35, 293-320, 1994.

Baggaley, W. J. and Galligan, D. P.: Mapping the interstellar dust flow into the Solar System, in Meteoroids 2001, edited by Warmbein, B., ESA SP-495, 663-669, 2001.

Benzenberg, J. F. and Brandes, H. W.: Versuch die Entfernung, die Geschwindigkeit und die Bahn der Sternschnuppen zu bestimmen, Annalen der Phys.,6, 224-232, 1800.

Betlem, H., ter Kuile, C. R., de Linge, M., van't Leven, J., Miskotte, K., and Jenniskens, P.: Precision meteor orbits obtained by the Dutch Meteor Society Photographic Meteor Survey (19811993), Astro. Astrophys. Supple. Ser., 128, 179-185, 1997.

Breit, G. and Tuve, M. A.: A radio method of estimating the height of the conducting layer, Nature, 116, 357, 1925.

Breit, G. and Tuve, M. A.: A test of the existence of the conducting layer, Phys. Rev., 28, 554-575, 1926.

Clegg, J. A., Hughes, V. A., and Lovell, J. C. B.: The Daylight Meteor Streams of 1947 May-August, Mon. Not. R. Astr. Soc., 107, 369-378, 1947.

Davies, J. G. and Gill, J. C.: Radio Echo measurements of the orbits of faint sporadic meteors, Mon. Not. R. Astr. Soc., 121, 437-462, 1960.

Elkin, W. L.: Photographic observations of the Leonids at the Yale Observatory, Ap. J., 9, 20-22, 1899.

Elkin, W. L.: The Velocity of Meteors as Deduced from Photographs at the Yale Observatory, Ap. J., 12, 4-7, 1900.

Ellyett, C. D.: The daytime meteor streams of 1949: measurement of velocities, Mon. Not. R. Astr. Soc., 109, 359-364, 1949.

Ellyett, C. D. and Davies, J. G.: Velocities of meteor measured by Diffraction of Radio Waves from Trails during formation, Nature, 161, 596-597, 1948.

Hasegawa, I.: Historical records of meteor showers, in Meteoroids and their parent bodies, edited by S̆tohl, J. and Williams, I. P., Slovak Academy of Sciences, Bratislava, 209-223, 1993.

Herlofson, N.: The theory of meteor ionization, Proc. Phys. Soc., 11, 444-445, 1946.

Hey, J. S.: ADRDE(ORG) Memorandum J4, AORG Report No. $275,1942$.

Hey, J. S. and Stewart G. S.: Derivation of meteor stream radiants by radio reflex methods, Nature, 158, 481-483, 1946.
Hey, J. S. and Stewart, G. S.: Radar observations of meteors, Proc. Phys. Soc., 59, 858-883, 1947.

Hey, J. S., Parsons, S. J., and Stewart, G. S.: Radio observations of the Giacobinid Meteor shower, 1946, Mon. Not. R. Astr. Soc., 107, 176-183, 1947.

Janches, D., Mathews, J. D., Meisel, D., and Zhou, Q.: Orbital properties of the Arecebo micrometeorites at Earth intersection, Icarus, 150, 206-218, 2001.

Le Verrier, U. J. J.: Sur les etoiles filantes de 13 Novembre et du 10 Aout, Comptes Rendus, 64, 94-99, 1867.

Lovell, A. C. B., Banwell, C. J., and Clegg, J. A.: Radio Echo Observations of the Giacobinid Meteors 1946, Mon. Not. R. Astr. Soc., 107, 164-175, 1947.

McCrosky, R. E. and Posen, A.: Orbital elements of photographic meteors, Smithson. Contr. Astrophys., 4, 15, 1961.

McKinley, D. W. R. and Millman, P. M.: A phenomenological theory of radar echoes from meteors, Proc. Inst. Radio Engrs., 37, 364-375, 1949.

Molau, S.: The AKM Video Meteor Network, in Meteoroids 2001, edited by Warmbein, B., ESA SP-495, 315-318, 2001.

Nagaoka, H.: Possibility of the Radio Transmission being disturbed by Meteoric Showers, Proc. Imp. Acad. Tokyo, 5, 233-236, 1929.

Öpik, E. J.: Note on stellar perturbations of nearly parabolic orbits, Proc. Amer. Acad. Arts and Sci., 67, 169-183, 1932.

Öpik, E. J.: Results of the Arizona expedition for the study of meteors V; on the distribution of heliocentric velocities, Harv. Coll. Obs. Circ., 391, 1934.

Öpik, E. J.: Analysis of 1436 meteor velocities, Pub. Tartu. Obs., 30, 86, 1940.

Öpik, E. J.: The failures, Irish Astron. Jl., 9, 156-160, 1969.

Pecina, P., Koten, P. and Stork, R.: Simultaneous optical and radar observations of meteors: another criterion of commonness, in Meteoroids 2001, edited by Warmbein, B., ESA SP-495, 425428, 2001.

Pellinen-Wannberg, A.: The high power large aperture radar method for meteor observations, in Meteoroids 2001, Ed Warmbein, B., ESA SP-495, 443-450, 2001.

Pellinen-Wannberg, A. and Wannberg, G.: Meteor observations with the European incoherent scatter UHF radar, J. Geophys. Res., 99, 11379-11390, 1994.

Porter, J. G.: An analysis of British meteor data, Mon. Not. R. Astr. Soc., 103, 134, 1943.

Schafer, J. P. and Goodall, W. M.: Observations of KennellyHeaviside Layer Heights During Leonid Meteor Shower of November 1931, Proc. Inst. Radio Engrs., 20, 1941-1945, 1932.

Schiaparelli, G. V.: Sur la relation qui existe entre les cometes et les etoiles filantes, Astronomische Nachrichten, 68, 331-332, 1867.

Shapley, H. Öpik, E. J., and Boothroyd, S. L.: The Arizona expedition for the study of meteors, Proc. Nat. Acad. Sci., 18, 16-23, 1932.

Skellett, A. M.: The effect of Meteors on Radio transmission through the Kennelly-Heavyside Layer, Phys. Rev., 37, 1668, 1931.

Skellett, A. M.: The ionizing effect of Meteors in Relation to Radio Propagation, Proc. Inst. Radio Engrs., 20, 1933-1941, 1932.

Skellett, A. M.: The ionizing effect of Meteors, Proc. Inst. Radio Engrs., 23, 132-249, 1935. 
Spurneý, P.: Exceptional fireballs photographed in central Europe during the period 1993-1996, Planetary \& Space Sci, 45, 541$556,1997$.

Taylor, A. D., Baggaley, W. J., and Steel, D. I.: Discovery of interstellar dust entering the Earth's atmosphere, Nature, 380, 323325, 1999.

Ueda, M., Fujiwara, Y., Sugimoto, M., and Kinoshita, M.: Results of double station TV observations in 1998 and 1999, in Meteoroids 2001, edited by Warmbein, B., ESA SP-495, 325-330, 2001.

Von Niessl, G. and Hoffmeister, C.: Katalog der Bestimmungsgrößen für 611 Bahnen großer Meteore, Denksch. d'Akad. d'Wiss. Wien, 10), 1925.
Weinek, L.: Astronomische Beobachtungen an der K.K. Sternwarte zu Prag, in den Jahren 1885, 1886, und 1887, 1886.

Whipple, F. L.: Photographic meteor studies I, Proc. Am. Phil. Soc., 79, 499-548, 1938.

Whipple, F. L.: Photographic meteor studies II: non-linear trails, Proc. Am. Phil. Soc., 82, 275-290, 1940.

Zhou, Q., Tepley, C. A., and Sulzer, M. P.: Meteor observations by the Arecebo $430 \mathrm{MHz}$ incoherent scatter radar I: results from time-integrated observations, J. Atmos. Terr. Phys., 57, 421-431, 1995. 